\title{
From Minitrack to NAVSTAR: The Early Development of the Global Positioning System, 1955-1975.
}

\author{
Leo B. Slater \\ Naval Research Laboratory, Washington, DC.
}

\begin{abstract}
The paper outlines the early days of satellite navigation, focusing on the work of the Naval Research Laboratory (NRL) on systems that contributed to the later Global Positioning System (GPS). NRL's innovations included the Minitrack system for space surveillance, the development of high-precision timing systems for use in space, the Timation navigation satellite program, and the first two Navigation Technology Satellites (NTS-1 and NTS-2). NAVSTAR-GPS emerged as a joint Department of Defense (DoD) program in 1973, by which time much of the GPS concept had been developed and a number of GPS technologies had been tested.
\end{abstract}

Index Terms - Global Positioning System, history, navigation, satellite ground stations, satellite navigation systems.

\section{INTRODUCTION}

There is a saying that success has many fathers; and GPS has certainly been a success. Today, GPS uses satellite-borne clocks to provide high-precision time-signals. These signals allow us to locate ourselves almost anywhere on Earth and are employed to synchronize a wide-range of communication systems. The system's components include satellites (and their launchers), ground stations, and user equipment (receivers). Those who have created all these elements and made them function together-GPS's many parents-include the DoD (especially the Navy and the Air Force) and numerous firms and contractors. This paper looks at the contributions of the Naval Research Laboratory (NRL).

The Naval Research Laboratory is the Navy \& Marine Corps' corporate research laboratory. It was founded in 1923 and in its early days focused primarily on radio and sonar research and development. A pioneering institution in space science and technology, NRL explored the edge of space with captured V-2 rockets in the 1940s and quickly moved on to design and build its own launch vehicles, telemetry systems, and scientific payloads.

In satellite navigation's early days, even before the term Global Positioning System had been coined, NRL pioneered a number of key innovations, including the use of clocks and time-signals from space for navigation. By the early 1970swhen NAVSTAR-GPS emerged as a joint DoD program under Air Force management - much of the GPS system had been conceptualized and its requirements defined. At NRL, researchers had built and flown two successful experimental navigation satellites, Timation I \& II, and were well along with Timation III, a satellite renamed and partially retasked as the (joint-services) Navigation Technology Satellite 1.

\section{NRL IN SPACE}

NRL's fundamental contributions to GPS began in the 1950s. In the run-up to the International Geophysical Year (IGY) in 1957-1958, NRL expanded its rocket-based space science programs and developed boosters for placing a research satellite in orbit. (The IGY also set the stage for the USSR's launch of Sputnik.) Following on its Viking Rocket Program, NRL proposed the Vanguard system for the U.S. IGY program. The 1955 NRL proposal, "A Scientific Satellite Program," [1] yielded three interconnected programs under Project Vanguard: an artificial satellite; a rocket booster; and a satellite tracking system, Minitrack.

Even at this stage, NRL researchers were thinking in terms of using a satellite to precisely measure the location of points on the Earth: "One useful function that can be served by the first artificial satellite lies in the field of geodesy. The United States has been and is now expending considerable effort and funds to refine geodetic measurements. One of the most difficult geodetic tasks involves tying together the various continental grids and locating the many islands with respect to these grids. Whereas it is possible to map entire continents by well-known methods of triangulation, the technique fails when it must be extended across large bodies of water. As a result, geodesists have turned to other methods which are, in general, based on the use of the only presently available satellite, the moon, as a measuring vehicle. Both the Army Map Service and the Naval Observatory are active in the use of the moon as a reference body for geodetic and time measurements...An artificial satellite, which would be small in size and mass, and at a short distance from the earth, would have numerous advantages over the moon..." [1] Using the moon for such a purpose is relatively easy if the weather is good and the moon is up. Such optical methods - directly observing the satellite or viewing it by telescope - would be the simplest way to use the satellite for navigation. But this had many drawbacks for a small, fast-moving object in space.

To move beyond optical methods, the NRL proposal also grasped the value of radio transmission in precision satellite tracking: "It appears highly desirable, therefore, to have some other method of observation. Such a method called Minitrack 
has been proposed by the Naval Research Laboratory...It depends upon radio interferometry and involves carrying a small transmitter in the satellite. The Laboratory has proven this technique experimentally in its ballistic missile research. It will provide precise observation of the satellite once every orbit at every station, day or night, fair weather or foul." [1] With a radio transmitter in orbit, satellite navigation could be made quite robust. And NRL had already developed the basic system for making such observations and had deployed it at the White Sands missile range in New Mexico.

Following on the 1955 proposal, NRL developed the Minitrack constellation of ground stations. Minitrack, too, had its roots in NRL's Viking program. "The Minitrack System is a radio tracking system for use with a satellite vehicle in an orbit of between 200 and 1200 miles altitude. It is an outgrowth of several phase-comparison angle-tracking systems built and field tested by NRL for application to ballistic missile guidance using the Viking Rocket. Except for operating frequencies and antenna configurations, the Minitrack System will be essentially identical to these systems...The complete Minitrack System will provide three coordinates of satellite position and three vectors of satellite velocity, plus accurate time of transit at each ground station once per revolution of the satellite. A simplified version of these ground stations will provide time of transit, east-west and north-south angular position, and angular velocity with respect to the ground station. Inasmuch as radio transmission from a sub-miniature transmitter in the satellite vehicle is utilized, operation is independent of time of day and of all normal weather conditions, excepting severe local thunderstorms. The Minitrack System will thus provide complete tracking and position information from the satellite to the limits of its accuracy and operating life." [1] Minitrack was up and running with no orbital transmitter to track until Soviet launch of the first artificial satellite, Sputnik, in 1957.

Minitrack - with sites around the world - tracked satellites by their continuous-wave output signals. The sites employed multiple antennas arranged in an interferometer configuration so that the angle of arrival of the signal could be determined. As the 1955 NRL proposal put it: "Basically, a phase comparison angle tracking system determines the angle of arrival of a radio signal by measuring the difference in length between two radio paths from the signal source in the satellite to each of two receiving antennas located a known distance apart. By virtue of the method of comparison, this path length difference can be measured to very small fractions of the radio wavelength used." [1] At the dawn of the space age, this system tracked the Sputnik satellites, Explorer 1, and NRL's Vanguard satellites.

The Vanguard 1 satellite was put into orbit on 17 March 1958. Its transmitter was powered by the first solar cells placed in orbit. It transmitted for some six years. With the establishment of NASA in 1958, the Vanguard program passed from NRL to the new space agency. [2]
Minitrack performed if the orbiting vehicles emitted their own signals, but an upgrade would be needed to detect and track non-radiating objects in space. This was NRL's Space Surveillance project, developed in the period of 1958 to 1964 and covering the continental U.S. (In 1961, the system became an independent operational command and has been tracking debris, satellites, etc. ever since.) In its initial conception, the system was a "fence" of radio energy extending up into space. NRL designed it to provide first pass detection of all space objects whose orbits crossed the fence. The first Space Surveillance experiment was performed by modifying the Fort Stewart Minitrack station (in Georgia) with longer antennas that were placed perpendicular to the Minitrack antennas. Another transmitter was moved to Alabama where it was fed into a 400 foot-long antenna. The pattern of this antenna was oriented to coincide with the pattern at Fort Stewart so any satellite appearing in the transmitting beam would reflect signals into the receiving beam. This experiment successfully demonstrated both the detection and orbit determination of the early satellites, so the full fence was then deployed as a series of transmitters and receivers arrayed across the southern tier of the U.S., located along a great circle. This version of the fence was a multi-static continuous wave radar with three transmitting stations and six interferometric receiving sites. Any radio-reflective object breaking the fence was detected and catalogued.

Though the fence could detect, locate, and obtain the direction to a satellite's position, it needed at least two passes to determine the orbit. This could take hours, depending upon the orbital period of the satellite and its orbital orientation to the fence. To establish orbits on a single pass, NRL deployed a second fence, behind the first. As initially constituted, the second fence was reading over-the-horizon signals that proved quite noisy. To reduce such noise, the transmitter and receiver were controlled by an atomic clock (cesium beam). This yielded a system with very precisely matched frequencies and two time standards that had to be synchronized by a third standard which was shifted back and forth between sites. Thus, high precision timing was added to ground stations for space surveillance.

\section{SATELLITE NAVIGATION AND TIMATION}

From these roots came insights in satellite tracking that led logically to satellite navigation. Both the Navy's Transit satellite navigation system and NRL's Timation satellites emerged from satellite tracking and space surveillance systems. Transit, developed by the Applied Physics Laboratory at Johns Hopkins University, employed the Doppler shift of satellite transmissions. This yielded twodimensional position data. Transit was rapidly developed and deployed with particular effect for ships to fix their positions at sea-very useful for nuclear submarines deployed far from their home ports. Timation was designed to give faster, three- 
dimensional positions through a signal system employing side tone ranging and time transfer.

Key to Timation and to GPS was the use of satellite-borne clocks. This innovation was based on the insight that took the high-precision clocks of satellite tracking stations from the ground and placed them on the satellites in orbit. This is one of several seminal technologies that NRL developed in the period before the GPS program was established. The idea for using a side tone ranging technique originated with Roger Easton in April 1964. Easton was a major contributor to Timation and other NRL space programs. Combined with celestial navigation techniques, side tone ranging from satellites proved a simple and accurate method for navigation, as well as for time determination. The translation of celestial navigation techniques - of the type practiced by sailors using sextants to plot the positions of stars-was first developed for the artificial satellites in December 1965. The Timation satellites would beam time signals to user receivers on the ground.

Critical to this new system were passive ranging and the transfer of time signals between orbiting satellites and ground stations. The ground stations tracked the satellite and determined its precise orbit. Passive ranging meant that the user needed only a receiver for obtaining the time signals of passing satellites and need not transmit anything to the satellite. Position and time corrections could be dealt with from a handful of ground stations. As Roger Easton wrote in 1967: "Consider synchronized clocks at two separated positions with hands that rotate in milliseconds or microseconds. Observer A is able to see B's clock visually. When he looks at B's clock he notices that it lags his own due to the time it takes for light to travel from B to A. The difference in time is proportional to the distance between the two clocks...If A does not know whether or not the clocks are synchronized, he must measure the distance to find out. When we correct for the error in synchronization the readings will give the range without $A$ transmitting to $B$. The lack of required transmission results in the technique being rated as a passive system." [3]

On 31 May 1967, a Delta-Agena booster carried Timation I into a 500 nautical mile circular polar orbit. This first Timation satellite was small and gravity-gradient-stabilized. Providing thirty-two channels of telemetry. For time keeping, Timation I carried a quartz crystal standard. NRL's Timation program also included work on receiver systems and ground tracking. A range of vehicles (small boats, aircraft, and trucks) successfully navigated by receiving Timation I signals. This was all done by passive ranging to the orbiting satellite. Timation I was also used to transfer time between ground stations with accuracies consistently below one microsecond.

On 30 September 1969, Timation II-designed and built by NRL, like its predecessor-was launched. The new satellite was equipped with an improved quartz oscillator and an electrical system that allowed for continuous operation. It also transmitted at two frequencies, 150 and $400 \mathrm{MHz}$. This differential allowed for the operators on the ground to correct for signal refraction by the ionosphere. Overall, the NRL team continued their development of onboard clocks, satellite systems, tracking and ground stations, and user receivers. Timation II proved a number of principles of satellite navigation: “(a) a navigator can determine his position and synchronize his clock by using the passive ranging technique, (b) the measured range data may be used to obtain a fix regardless of whether the user's frequency is known or unknown, and (c) the time difference may be obtained in either case..." [4] Timation II clearly demonstrated the enormous potential of satellite navigation using orbital clocks and passive ranging.

During the late 1960s, the individual armed services and the Department of Defense exchanged information on an ongoing basis. And NRL's Timation program continued to evolve, as proposed in 1971: "The Timation Satellite System employs a constellation of 27 satellites - each in an 8-hour, 55-degree inclined orbit, ground stations in the vicinity of the Samoan Islands, the Virgin Islands, Guam, and central Alaska, and a variety of user equipments which are graded in cost and performance according to the user's requirements. This system will provide global, all-weather, passive, continuous navigation fixes in three dimensions with accuracies better than 50 feet. Low-cost equipment for general navigation users, at reduced accuracies, will be available for use with the system." NRL continued to learn from its ongoing R\&D and its two earlier Timation satellites. In 1971, the Laboratory began building a third satellite in this series. As enumerated in the "Timation Development Plan," the ground stations had an ongoing role, fulfilling the following functions:

1. Track the satellites.

2. Preprocess the received tracking data and prepare it for transmission to the central ground station.

3. Correct the epoch of the local time standard by

comparing it with the system standard via a covisible satellite.

4. Transmit data to, and receive from, the central ground station via a covisible satellite.

5. Correct the clock errors of all satellites in view as necessary.

6. Monitor the navigation signals. [5]

The proposed satellites would test new timekeeping devices, orbital configurations, and continue to develop the Timation system in line with the Joint Chiefs of Staff specifications and requirements.

Of course the Navy-with Transit and Timation-was not alone in having an interest in satellite navigation. Other services within the Department of Defense had programs and needs. DoD's higher echelons sought efficiencies and cost savings through the coordination of these resources and requirements. As early as 1968, a special study panel completed a review of military navigation, and the Joint 
Chiefs of Staff issued new specifications for a Department of Defense system. From this joint initiative came, in due course, a Navigation Satellite Executive Steering Group, a Defense Navigation Satellite Development Program, and eventually a joint program under Air Force management: In 1973, the Department of Defense established the NAVSTAR-GPS Program. [6]

The Navigation Technology Satellites (NTS) were the first hardware in the NAVSTAR-GPS series. NTS-1 was, in fact, NRL's Timation III satellite, launched 14 July 1974-bearing both designations - and carrying not just an improved signal package from NRL but also an experimental System 621B transmitter from the earlier Air Force program. Organizationally, the marriage of the Air Force 621B program with NRL's Timation program was the critical convergence that led to today's GPS. On the technology side, it should also be noted that Timation III/NTS-1 carried an atomic clock in the form of two experimental rubidium standards. [7] As the Air Force's Col. B. W. Parkinson and his coauthor later wrote: "development of a reliable, ultra-stable, space-qualified atomic frequency standard became one of the pacing development items for the GPS program." [6]

Launched on 23 June 1977, NTS-2 was the first satellite designed, built, and launched in the joint NAVSTAR-GPS program. It was built by NRL. As with NRL's earlier navigation satellites, new systems and techniques were tested with each spacecraft. NTS-2's primary solar arrays were new, high-efficiency cells employing the sun-orienting mechanism later used on the NAVSTAR satellites. Another innovation tested on NTS-2 was the use of nickel-hydrogen batteries in support of the solar arrays. This was a cooperative experiment with INTELSAT who provided the battery cells, while NRL integrated them into satellite power systems. [8]

NRL continued to make other major contributions in engineering. For example, NRL worked with the contractor, Fairchild, on the upper stage of the Atlas F booster that carried NTS-2 into orbit. The same upper stage carried the Block I NAVSTAR satellites precisely into the GPS orbit. Such an orbital insertion was at the time quite a feat. The NRL teamheaded by Peter Wilhelm-provided key innovations. Each GPS spacecraft had to be inserted into its assigned position in the constellation, first launching the vehicle into a higheccentricity transfer orbit and then pushing it into a loweccentricity drift orbit. Such engineering inputs made GPS a viable technology.
With the transition from the Navigation Technology Satellites to the NAVSTAR-GPS program, NRL would continue to provide critical technical support and key innovations even as the program grew in scale and complexity and as the range of users and uses grew tremendously. Today, GPS is a free utility that has given rise to a host of technologies and applications. Without the pioneers of Minitrack and Timation, this powerful technological system might never have been born. The Navy's in-house R\&D capabilities allowed it to innovate. And NRL's deep traditions of radio research and space science $\&$ technology underpinned its development of satellite navigation. Timation/GPS illustrates how NRL independently advanced DoD science \& technology in an area of little or no academic or industrial interest. Through the years, NRL has maintained broad expertise in satellites and their associated technologieslargely housed today in NRL's Naval Center for Space Technology. [9]

\section{REFERENCES}

[1] Rocket Development Branch Rocket Sonde Branch, Atmosphere \& Astrophysics Division Electron Optics Branch, and Optics Division, "A Scientific Satellite Program," NRL Memorandum Report no. 487, 5 July 1955.

[2] C. M. Green and M. Lomask, Vanguard: A History, Washington DC: NASA, 1970.

[3] R.L. Easton, "Space Applications Branch Technical Memo \#1," 8 May 1967.

[4] T.B. McCaskill, J.A. Buisson, and D.W. Lynch, "Principles and Techniques of Satellite Navigation Using the Timation II Satellite," NRL Report no. 7252, 17 June 1971.

[5] Space Applications Branch Space Technology Division, "Timation Development Plan," NRL Report no. 7227, 2 March 1971.

[6] B. W. Parkinson and S. W. Gilbert, "NAVSTAR: Global Positioning System-Ten Years Later," Proc. IEEE, vol. 71, No. 10, pp. 1177-1186, October 1983.

[7] T. B. McCaskill and J. A. Buisson, "NTS-1 (Timation III) Quartz and Rubidium Oscillator Frequency Stability Results," Proc. of the IEEE 29th Annual Symposium on Frequency Control, May 1975, 1537055, pp. 425-435.

[8] R.L. Easton, J.A. Buisson, T.B. McCaskill, O.J. Oaks, S. Stebbins, and M. Jefferies, "The Contribution of Navigation Technology Satellites to the Global Positioning System," NRL Report no. 8360, 28 December 1979.

[9] For more on NRL and GPS, see: R.R. Whitlock and T.B. McCaskill, "NRL GPS Bibliography," NRL Memorandum Report no. 1001-09-8999, 3 June 2009. 